\title{
A Case of Intradural Extramedullary Spinal Tuberculosis Diagnosed 8 Years After Treatment of the Primary Infection
}

\author{
Kushagra Verma, MD \\ Thomas Jefferson University \\ Tracey Crothers, MD \\ University of Southern California \\ Brian Neuman, MD \\ Thomas Jefferson University \\ Alexander Vaccaro, MD, PhD \\ Thomas Jefferson University \\ Joshua Heller, MD \\ Thomas Jefferson University
}

Follow this and additional works at: https://jdc.jefferson.edu/jhnj

\section{Let us know how access to this document benefits you}

\section{Recommended Citation}

Verma, MD, Kushagra; Crothers, MD, Tracey; Neuman, MD, Brian; Vaccaro, MD, PhD, Alexander; and Heller, MD, Joshua (2013) "A Case of Intradural Extramedullary Spinal Tuberculosis Diagnosed 8 Years After Treatment of the Primary Infection," JHN Journal: Vol. 8 : Iss. 1 , Article 4.

DOI: https://doi.org/10.29046/JHNJ.008.1.005

Available at: https://jdc.jefferson.edu/jhnj/vol8/iss1/4

This Article is brought to you for free and open access by the Jefferson Digital Commons. The Jefferson Digital Commons is a service of Thomas Jefferson University's Center for Teaching and Learning (CTL). The Commons is a showcase for Jefferson books and journals, peer-reviewed scholarly publications, unique historical collections from the University archives, and teaching tools. The Jefferson Digital Commons allows researchers and interested readers anywhere in the world to learn about and keep up to date with Jefferson scholarship. This article has been accepted for inclusion in JHN Journal by an authorized administrator of the Jefferson Digital Commons. For more information, please contact: JeffersonDigitalCommons@jefferson.edu. 


\section{A Case of Intradural Extramedullary Spinal Tuberculosis Diagnosed 8 Years After Treatment of the Primary Infection}

\author{
Kushagra Verma, MD'; Tracey Crothers, $\mathrm{MD}^{2}$; Brian Neuman, MD; \\ Alexander Vaccaro MD, $\mathrm{PhD}^{4}$; Joshua Heller, $\mathrm{MD}^{5}$ \\ 'PGY3, Department of Orthopaedics, Thomas Jefferson University Hospital, Philadelphia, PA \\ ${ }^{2}$ PGY1, Department of Orthopaedic Surgery, University of Southern California, Los Angeles, CA \\ ${ }^{3}$ Chief Resident, Department of Orthopaedics, Thomas Jefferson University Hospital, Philadelphia, PA \\ ${ }^{4}$ Professor and Attending Surgeon of Orthopaedics and Neurological Surgery, Thomas Jefferson \\ University Hospital, Vice Chairman, Department of Orthopaedics, Philadelphia, PA \\ ${ }^{5}$ Assistant Professor, Department of Neurological Surgery, Thomas Jefferson University \\ Hospital and Jefferson Hospital for Neuroscience, Philadelphia, PA
}

\section{INTRODUCTION}

Tuberculosis (TB), the disease caused by Mycobacterium tuberculosis, is the second leading killer worldwide from a single infectious agent. Spinal TB is associated with pulmonary disease and may originate from (1) hematogenous spread outside the central nervous system (CNS), (2) sites located within the cranium (meningitis), or (3) secondary extension from vertebral bodies or discs (Pott's disease). ${ }^{1}$

Other presentations of tuberculosis include tuberculous arachnoiditis, nonosseous spinal tuberculoma, and spinal meningitis. Of these conditions, spinal tuberculoma can be intradural, extradural, intramedullary, or extramedullary. Intradural extramedullary tuberculosis is the most rare type and is only observed in 1 out of 50,000 cases of tuberculosis. ${ }^{2,3}$ To date, there has only been one documented intradural extramedullary tuberculoma in a non-HIV-infected patient in North America. ${ }^{4}$ Intradural spinal tuberculomas often present simultaneously or are preceded by tuberculous meningitis (TBM) ${ }^{5,6}$
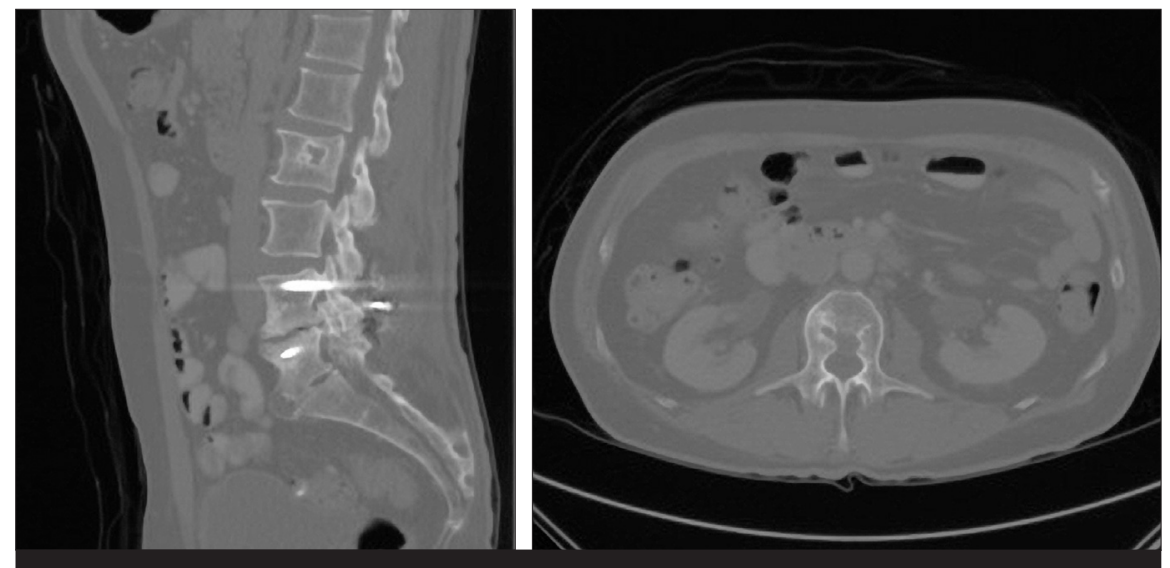

Figure 1

Preoperative sagittal (a) and axial (b) CT images at the L2 level shows a lytic lesion with a scleroitic border. Hardware from a previous fusion is also noted at the L4-L5 level.
We treated an otherwise healthy 68-yearold male that developed progressive lower extremity pain and weakness from an intradural spinal tuberculoma. While spinal tuberculomas have been previously described, no prior author has reported a tuberculoma several years after successful eradication of the pulmonary infection. In this case report, we present the case, pertinent imaging, operative findings, histopathology, and a review of the literature.

\section{CASE DESCRIPTION}

A 68-year-old male presented with a 4-month history of progressive low back pain, left lower extremity weakness, and radiculopathy (pain 7 out of 10) worsened with activity. There was no history of recent trauma, bowel or bladder incontinence, balance problems, neck pain, or upper extremity radiculopathy.

Past medical history was significant for diabetes mellitus, diabetic neuropathy, and tuberculosis diagnosed in 2004 following an excisional biopsy of a right lung mass. At that time, he was treated with Rifampin, Isoniazid, Pyrazinamide, and Ethambutol (RIPE). Surgical history included an L4-L5 discectomy in 1984, revision discectomy in 1990, and a L4-5 decompression and fusion in 2006. He has a 35 pack per year smoking history, drinks socially, is retired and lives with his family.

Neurologic examination revealed decreased left lower extremity strength: $2 / 5$ iliopsoas, $3 / 5$ quadriceps, $4 / 5$ hamstring, $2 / 5$ tibialis anterior, 4/5 extensor hallicus longus, and 4/5 gastrocnemius. His sensation to light touch was decreased on the anterior and lateral aspects of the left leg compared to the right. The patellar and achilles reflex were absent on the left, and 1+ in all other distributions. The remainder of his neurologic evaluation and physical exam were unremarkable. He was afebrile with stable vital signs on admission. The laboratory values were as follows: WBC 

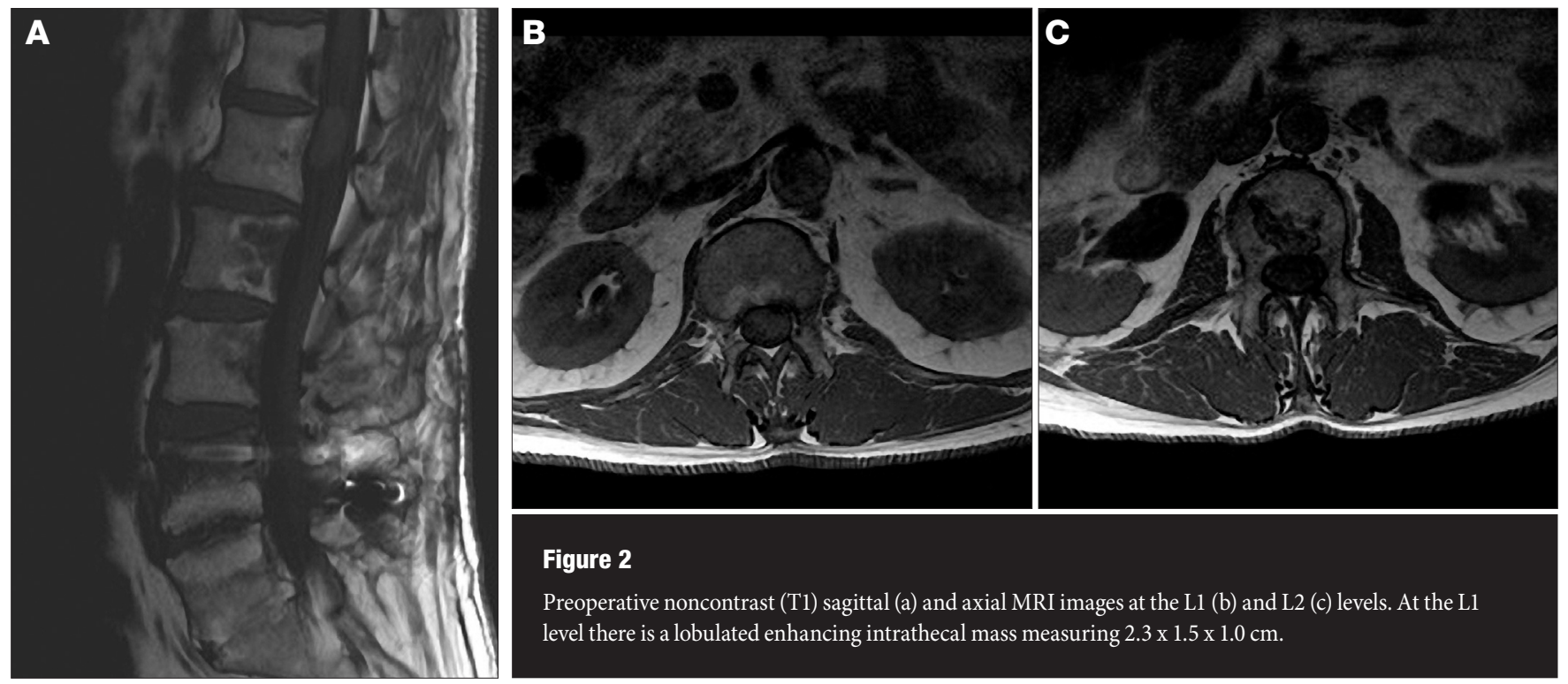

\section{Figure 2}

Preoperative noncontrast (T1) sagittal (a) and axial MRI images at the L1 (b) and L2 (c) levels. At the L1 level there is a lobulated enhancing intrathecal mass measuring $2.3 \times 1.5 \times 1.0 \mathrm{~cm}$.

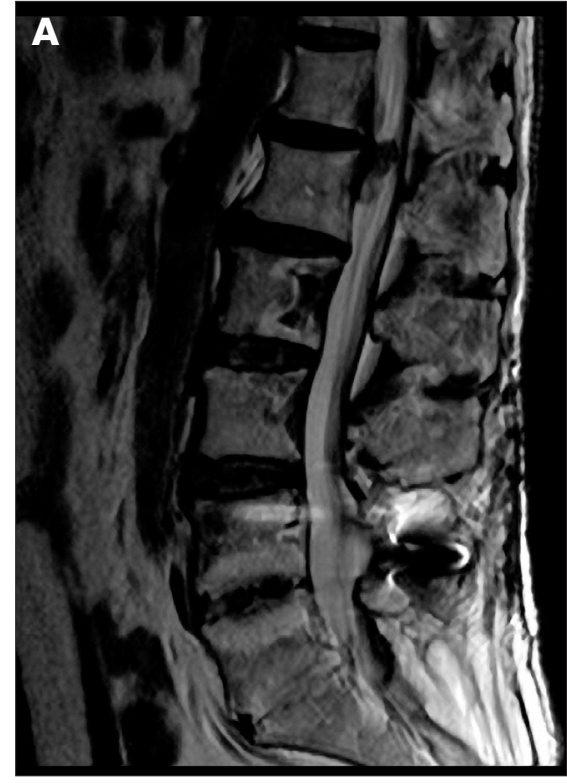

of 8.3, slightly elevated ESR of 25, CRP of 1.60 , transferrin of 262 , and an albumin of 4.0 .

A computerized tomography $(\mathrm{CT})$ of the chest revealed a $7.7 \times 3.6 \mathrm{~cm}$ irregular mass in the right upper lobe. CT Imaging of the spine revealed lytic lesions in the posterior aspect of the L2 and L3 vertebral bodies, as well as scalloping of the L1 and L2 vertebral bodies (Figure 1). Magnetic Resonance Imaging (MRI) of the spine demonstrated a lobulated enhancing intrathecal mass at the level of L1 measuring $2.3 \times 1.5 \times 1.0 \mathrm{~cm}$ (Figure 2). Contrast enhanced images revealed increased
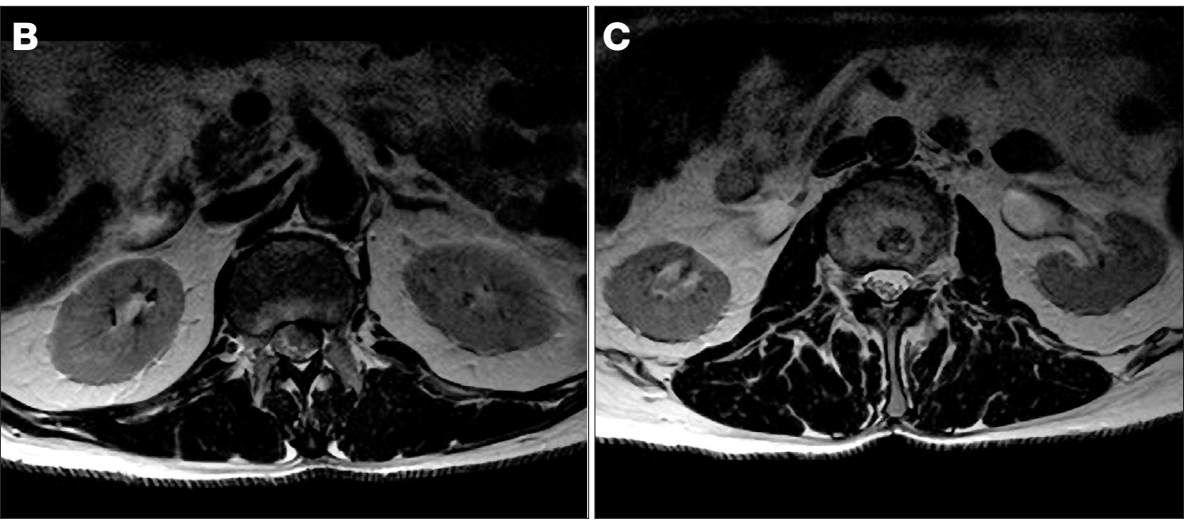

\section{Figure 3}

Preoperative contrast enhanced (T2) sagital (a) and axial MRI images at L1 (b) and L2 (c) respectively, demonstrating a lobulated enhancing intrathecal mass at the level of L1. There is signal abnormality noted to extend along the posterior aspects of the vertebral body from T12 to L2 and along the left neural foramen at T12-L1

signal along the posterior aspect of the L1-L3 vertebral bodies and along the left neural foramen of T12-L1 (Figures 3).

The patient was taken to the operating room for an emergent decompression of the intradural mass with extension to the left T12-L1 neural foramina. A medial facetectomy and bilateral laminectomy allowed visualization of the dural sac and ventrally located mass with a operating microscope. The mass was adherent to several nerve rootlets and exited left of the T12 nerve root (Figure 4a). There was notable nerve root clumping and arachnoiditis at the
T12/L1 level (Figure 4b). Surrounding the lesion an inflammatory capsule wall was identified. Scoring of the lesion resulted in an egress of purulent fluid. The lesion along with its extension to the nerve root sleeve was debulked. The region was copiously irrigated with saline solution and the dura was reapproximated and closed primarily.Multiple samples were sent for culture and pathology. Intraoperative bronchial brushings and biopsy revealed polymicrobial organisms. Biopsy of the right upper lung lobe showed mild chronic inflammation, but was negative for 

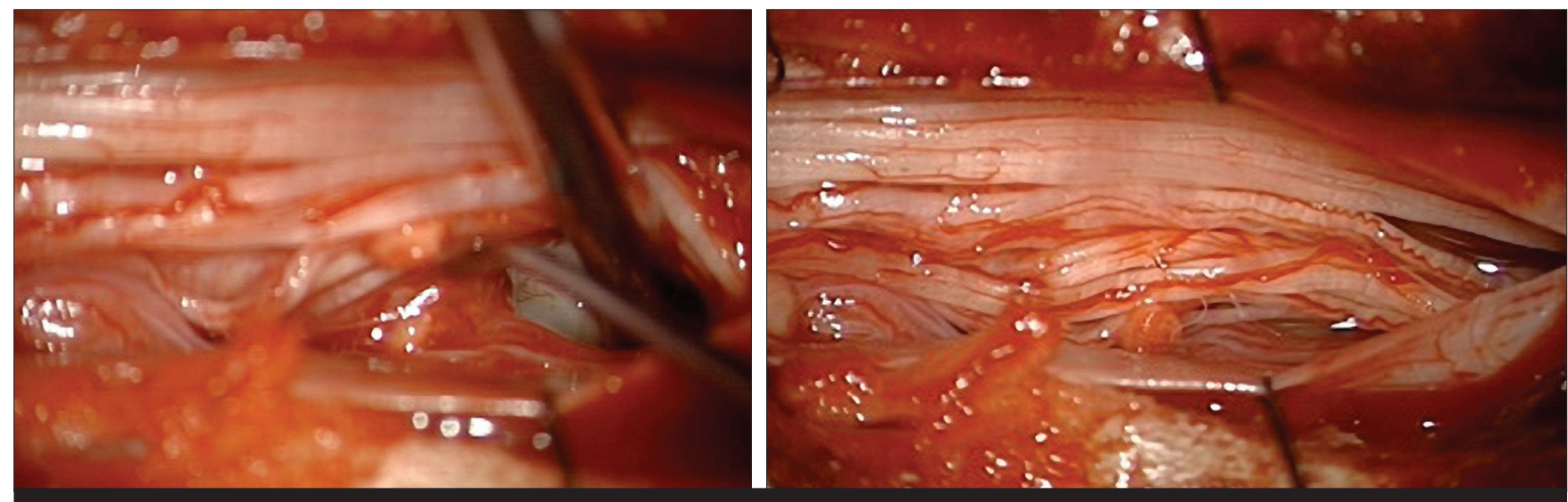

Figure 4

Intraoperative photographs showing an intradural mass compressing on the ventral nerve rootlets and could be seen exiting left of the T12 nerve root (a). After the mass is debulked, there is residual clumping of nerve roots in the cauda equina (b).

granulomas and malignancy. Frozen section of the intradural mass revealed a necrotizing inflammatory process with histiocytes, suggestive of tuberculosis. The mass was composed of dense connective tissue consistent with a portion of dura with focal necrosis and mixed chronic and acute inflammatory infiltrate. No acid-fast, fungal, or bacterial organisms were identified. Microscopically, small fragments of dura showed areas of necrosis and a mix of polymorphonuclear leukocytes, reactive T-lymphocytes, rare reactive $\mathrm{B}$-lymphocytes and small clusters of macrophages.

Post-operatively, the patient's hospital course was uncomplicated. He was empirically treated with RIPE and followed closely with MRI imaging which showed decreased enhancement at the T12/L1 level. At 8-month follow-up, he reported minimal pain, independent ambulation, and near complete resolution of his neurologic deficit. Physical exam revealed isolated weakness of his left tibialis anterior. ${ }^{4,5}$

\section{DISCUSSION}

Tuberculosis infection of the spine classically presents as Pott's disease. Other presentations include tuberculous arachnoiditis, nonosseous spinal tuberculoma, and spinal meningitis. In North America, Dibble et. al. was one of the first to describe a patient with progressive paralysis and intradural TB that developed after initiating medical treatment. Histology showed "areas of necrosis surrounded by granulomatous tissue", however acid-fast staining and intra-operative cultures were negative. ${ }^{7}$ From 1980 to 2005, only 22 cases of intradural extramedullary TB have been reported in the world. ${ }^{6}$ All of these cases were from outside North America primarily in young HIV-negative patients. To date, there has only been one reported case of intradural extrameduallary TB reported in North America in the last 30 years, our case being the second. ${ }^{4}$

Among adult patients with intradural extramedullary TB, there appear to be three variants: (1) healthy patients with no prior history of primary $\mathrm{TB}$, (2) patients with disseminated TB elsewhere in the body, (3) and those patients with a recent history of TB treated successfully with medical therapy. In the first group, acid-fast staining and operative cultures are positive and strongly suggestive of the disease. In our review, there have only been three case reports in this group..$^{8,4,2}$ The second group is the largest, and may include HIV positive patients on retroviral therapy. ${ }^{4,3}$ In contrast, patients in the third group paradoxically develop intradural pathology only after the initiation of anti-tuberculosis medications. ${ }^{4,3,9,6,10}$

Luo et al. reported a case of intradural TB in a HIV-negative patient with no known primary infection and no risk factors for exposure. ${ }^{4}$ Similarly, Compton et al. reported an unusual case of intradural tuberculoma in the cervical spine identified in a healthy HIV negative male after a trivial car accident.
The patient had no history of TB, but both parents had pulmonary TB. For both of these cases, histology revealed a granulomatous inflammatory mass. Acid fast staining and intra-operative cultures were positive. ${ }^{8,4}$ Similarly, Mirzai et al. reported intradural tuberculosis in a young healthy patient with no prior history of pulmonary TB. In this case, histology revealed granulomatous inflammation and giant cells, which were suggestive, however, acid-fast staining and intra-operative cultures were negative.

Among cases with a known history of pulmonary tuberculosis, O'Hickey et al. reported the formation of a spinal tuberculoma 15 months after successful treatment of pulmonary tuberculosis. ${ }^{9}$ Similarly, Shim et. al described a patient with intradural TB that developed only 5 weeks after initiation of medical treatment. ${ }^{11}$ For both cases, acid-fast stains and cultures were negative but histology confirmed a granulomatous inflammatory mass. In a review of 25 case reports by Roca et al., the formation of an intradural extramedullary mass seems to paradoxically form a few weeks after the initiation of anti-TB medications in young healthy HIV-negative patients. In almost all cases, acid-fast staining and cultures taken during surgical decompression are negative, while histology is suggestive. The mechanism is poorly understood, however interplay between the bacteria and the host's immune system may be responsible for this finding. ${ }^{4,6}$ Tuberculomas may also represent a granulomatous healing response rather than a true bacteriologic reactivation. ${ }^{9}$ 
The patient presented in this report developed lower extremity weakness and pain due to the formation of an intradural extramedullary tuberculoma. The insidious nature of his presentation, negative microbial studies, and granulomatous histology are typical for this disease. However, the 8-year delay between his pulmonary disease and subsequent spinal pathology is atypical and unreported. To our knowledge, he is also the oldest known patient to develop an intradural extramedullary tuberculoma. Lastly, this is only the second case of an intradural tuberculoma in a healthy patient from North America. This combination represented a diagnostic challenge for both orthopaedic and neurosurgeons. A detailed medical and travel history in conjunction with a high index of suspicion is necessary for the diagnosis.

\section{REFERENCES}

1. Turgut M. Spinal tuberculosis (Pott's disease): Its clinical presentation, surgical management, and outcome. A survey study on 694 patients. Neurosurg Rev. 2001;24:8-13.

2. Muthukumar N, Venkatesh G, Senthilbabu S, Rajbaskar R. Surgery for intramedullary tuberculoma of the spinal cord: Report of 2 cases. Surg Neurol. 2006;66(1):69-74; discussion 74. doi: 10.1016/j. surneu.2005.10.024.

3. Newton R. Tuberculous meningitis. Archives of Disease in Childhood. 1994;70:364-366.

4. Mirzai $\mathrm{H}$. Tuberculoma of the cervical spinal canal mimicking en plaque meningioma. J Spinal Disord Tech. 2005;18(2):197-199.

5. Kumar K. A clinical study and classification of posterior spinal tuberculosis. Int Orthop. 1985;9(3):147-152.

6. Skendros P, Kamaria F, Kontopoulos V, Tsitouridis I, Sidiropoulos L. Intradural, eextramedullary tuberculoma of the spinal cord as a complication of tuberculous meningitis. Infection. 2003;31(2):115-117.

7. Dibble J, Cascino J. Tuberculoma of spinal cord. JAMA. 1956;162(5):461-462.

8. Compton JS, Dorsch NW. Intradural extramedullary tuberculoma of the cervical spine. case report. J Neurosurg. 1984;60(1):200-203. doi: 10.3171/ jns.1984.60.1.0200.

9. Roca B. Intradural extramedullary tuberculoma of the spinal cord: A review of reported cases. J Infect. 2005;50(5):425-431. doi: 10.1016/j.jinf.2004.07.012.

10. Tang L, Swash M. Tuberculosis of the nervous system: a modern problem. Journal of the Royal Medicine. 1985;78:429-432.

11. Sree Harsha C, Shetty A, Rajasekaran S. Intradural spinal tuberculosis in the absence of vertebral or meningeal tuberculosis: a case report. Journal of Orthopaedic Surgery. 2006;14(1):71-75.

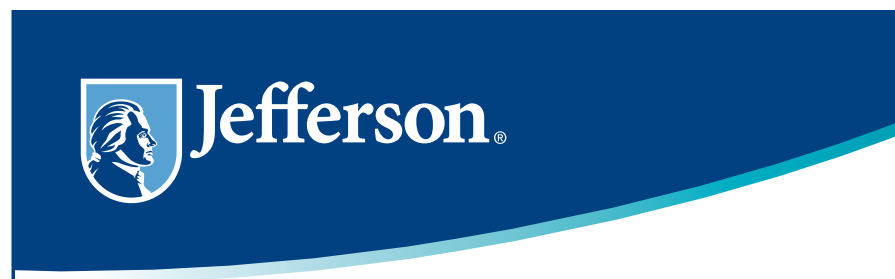

\section{Brain Tumor Support Group at Jefferson}

The group is open to patients with all types of brain tumors, along with their families. It offers a wonderful opportunity to meet others facing similar situations and feelings. The group conducts open meeting time for general support and networking, and it also features occasional guest speakers to enhance knowledge and understanding.

\section{Location}

Jefferson Hospital for Neuroscience (JHN)

3rd Floor Conference Room

900 Walnut Street, Philadelphia, PA 19107

\section{Date}

Second Thursday of every month, except for July and August

\section{Time}

7 - 8:30 p.m.

\section{Facilitator}

Alisha Amendt, MSN CRNP

\section{Contact}

phone: 215-955-4429

e-mail: alisha.amendt@jefferson.edu

\section{Parking}

Validated parking available for the Jefferson Hospital for Neuroscience (JHN) garage

\section{Other}

Light refreshments and snacks will be served 